\title{
Barreiras para a prática de atividade física em idosos: revisão de escopo de estudos brasileiros
}

\section{Barriers to physical activity in elderly: scoping review of Brazilian studies}

\section{AUTORES \\ Thiego da Silva Socoloski ${ }^{1}$ (D) \\ Cassiano Ricardo $\operatorname{Rech}^{2}$ (D) \\ Jaime Alves Correa Junior ${ }^{1}$ (D) \\ Rodrigo Meireles Lopes ${ }^{1}$ (D) \\ Adriano Akira Ferreira $\mathrm{Hino}^{3}$ (D) \\ Paulo Henrique Guerra ${ }^{1}$ (D) \\ 1 Universidade Federal da Fronteira Sul, Chapecó, Santa Catarina, Brasil. \\ 2 Universidade Federal de Santa Catarina, \\ Programa de Pós-Graduação em Educação Física, \\ Florianópolis, Santa Catarina, Brasil. \\ 3 Pontifícia Universidade Católica do Paraná, \\ Programa de Pós-graduação em Ciências da Saúde, \\ Curitiba, Paraná, Brasil.}

\section{CONTATO}

Paulo Henrique Guerra

paulo.guerra@ufs.edu.br

Rodovia SC 484, Km 02, Chapecó, Santa

Catarina, Brasil.

CEP: 89815-899.

DOI

$10.12820 /$ rbafs.26e 0208

\begin{abstract}
RESUMO
Com objetivo de identificar as barreiras à prática de atividade física $(\mathrm{AF})$ em idosos, foi conduzida uma revisão de escopo, com buscas em sete bases de dados eletrônicas e em listas de referências, abrangendo artigos disponíveis até dezembro de 2020. Procurou-se por estudos originais, desenvolvidos no Brasil, que investigaram barreiras à prática de $\mathrm{AF}$ em amostras de pessoas $\geq 60$ anos. Dos 911 artigos iniciais, 13 compuseram a síntese, envolvendo idosos participantes de grupos de convivência, institucionalizados e participantes de projetos de AF, com predominância de mulheres. Foram identificadas 31 barreiras, sendo 18 delas classificadas como intrapessoais $(58,1 \%)$. "Doença, dor ou lesão" foi a barreira mais frequentemente mencionada entre os estudos $(n=9)$, estando também associada à inatividade física em um artigo. Também merecem destaque: "falta de segurança" $(\mathrm{n}=6)$, "medo de cair/se machucar" ( $n=3)$ e "sentir-se suficientemente ativo" $(n=3)$. As barreiras intrapessoais são as mais percebidas pelos idosos brasileiros, sendo a barreira "dor, doença e/ou lesão" a mais frequentemente mencionada, seguida das barreiras "falta de segurança"; "medo de cair/se machucar" e "sentir-se suficientemente ativo". Mesmo que esta evidência esteja amparada, em sua maior parte, por dados provenientes da região Sul do país, o reconhecimento destas barreiras pode ser incorporada nos distintos cenários de oferta da AF para idosos. Complementarmente, futuros estudos são importantes, tanto para apresentação das principais barreiras para a $\mathrm{AF}$ nas distintas regiões do país, quanto para a análise das suas possíveis associações à inatividade física.
\end{abstract}

Palavras-chave: Atividade física; Idoso; Brasil; Revisão.

\section{ABSTRACT}

In order to identify barriers to physical activity $(P A)$ in the elderly, we conducted a scoping review, with searches in seven electronic databases and reference lists, covering articles available until December 2020. We searched for original studies, developed in Brazil, that investigated barriers to PA in samples of $\geq 60$ years people. Of the 911 initial results, 13 composed the synthesis, involving elderly participants of socialization groups, institutionalized and participants of PA projects, with a predominance of women. Thirty-one barriers were identified, 18 of which were classified as intrapersonal (58.1\%). "Disease, pain or injury" was the most frequently mentioned barrier among the studies $(n=9)$, being also associated with physical inactivity in one article. Also noteworthy were "lack of safety" ( $n=6)$, "fear of falling/injury" $(n=3)$, and "feeling active enough" $(n=3)$. Intrapersonal barriers are the most perceived by Brazilian elderly, with "pain, illness and/or injury" being the most frequently mentioned barrier, followed by "lack of safety", "fear of falling/injury", and "feeling active enough". Even though this evidence is mostly supported by data from the Southern region of the country, the recognition of these barriers can be incorporated into the different scenarios of PA supply for the elderly. In addition, future studies are important, both for the presentation of the main barriers to $P A$ in different regions of the country, and for the analysis of their possible associations with physical inactivity.

Keywords: Physical activity; Aged; Brazil; Review.

\section{Introdução}

Mesmo que ocorrendo de forma heterogênea entre as regiões do país, o processo de envelhecimento da população brasileira, datado desde a década de 1950, pode ser entendido como uma das consequências da transição demográfica ${ }^{1}$. Em vista das especificidades no perfil de doenças ${ }^{2}$, incapacidade $^{3}$ e das sequelas que estão associadas à esta faixa etária ${ }^{4}$, o envelhecimento da população brasileira é um dos principais desafios da saúde pública no país ${ }^{1}$. Nesse sentido, diante das demandas específicas das pessoas com idade mais avançada, estratégias e ações para a melhoria e manutenção da sua saúde fazem-se necessárias ${ }^{4,5}$.

Dentro destas, pode se destacar o papel benéfico da prática regular de atividade física (AF) em diversos domínios da vida de um idoso, como autonomia funcional ${ }^{6}$, função $\operatorname{cognitiva}^{7}$, menor risco de queda ${ }^{8}$, realização de atividades da vida diária ${ }^{4,9}$ e melhor qua- 
lidade de vida ${ }^{4,10}$. Entretanto, da mesma forma que se observa no contexto internacional ${ }^{11}$, idosos brasileiros também apresentam menores níveis de AF moderadas e vigorosas no tempo de lazer, quando comparados a outros grupos etários ${ }^{12}$, o que torna importante a identificação e o debate sobre as barreiras que impedem a prática de $\mathrm{AF}$.

Dentre as pesquisas conduzidas no país, uma revisão sistemática prévia reconhece distintas barreiras para a prática de AF nas diferentes fases da vida, nos domínios individual, contextual e ambienta ${ }^{13}$. Contudo, visando uma apresentação e discussão mais específica das barreiras relatadas pelos idosos, de modo que tal evidência possa ser incorporada nos distintos contextos de oferta da $\mathrm{AF}$ direcionados à esta faixa etária (e. g., aulas, conteúdos, políticas públicas), o presente trabalho teve como objetivo identificar as barreiras à prática de AF em idosos, considerando-se a evidência dos estudos conduzidos no Brasil.

\section{Métodos}

O presente trabalho configura-se como uma revisão de escopo, registrada na plataforma Open Science Framework (Título: Barriers to physical activity in Brazilian elderly: scoping review).

Como critérios de inclusão para a síntese, foram procurados artigos científicos originais que analisaram as barreiras para a prática de AF (ou inatividade física) em amostras de idosos que vivem no Brasil, com idade igual ou acima dos 60 anos, sem restrições quanto aos delineamentos de pesquisa utilizados, representatividade amostral, sexo (e. g., pesquisas com amostras apenas de mulheres), condição contextual e abordagens utilizadas para coleta e análise dos dados (e. g., qualitativas, quantitativas ou mistas). Em relação às características clínicas dos participantes, em um primeiro momento, optou-se por amostras heterogêneas, entretanto, a partir do reconhecimento de projetos conduzidos em amostras de idosos institucionalizados e/ou com diagnósticos de doenças crônicas não transmissíveis, em um segundo momento estes artigos foram revistos e também considerados para a síntese descritiva. Para uma melhor comparabilidade, foram procurados artigos que especificamente utilizaram o termo e instrumentos para identificação das "barreiras" para atividade física ou inatividade física.

Em sete de dezembro de 2020, os artigos científicos foram procurados por meio de buscas em sete bases de dados eletrônicas (Lilacs, Physical Education Index,
Pubmed, Scielo, Scopus, Sportdiscus e Web of Science) e por meio de buscas manuais nas listas de referências dos artigos avaliados pelos seus textos integrais. As estratégias foram elaboradas a partir da construção desenvolvida para o Pubmed: ((barrier*[Text Word]) AND ((((physical activity[Text Word]) OR walk*[Text Word]) OR run*[Text Word]) OR cycling[Text Word])) AND brazil[Text Word].

Esta revisão foi desenvolvida por meio de cinco etapas: (I) elaboração das buscas sistemáticas, (II) avaliação dos títulos e dos resumos, (III) avaliação dos textos integrais, (IV) extração dos dados originais e (V) desenvolvimento da síntese descritiva. A etapa I envolveu o trabalho conjunto de quatro pesquisadores (JC, PG, RM e TS); as etapas II, III e IV foram conduzidas por três avaliadores, de forma independente (JC, RM e TS), com auxílio de um quarto avaliador (PG) e a etapa $\mathrm{V}$ foi conduzida por dois pesquisadores, que também trabalharam de forma independente (PG e TS).

A extração dos dados originais foi feita em uma planilha eletrônica, dividida em informações descritivas (e. g., autor, local da pesquisa, amostragem, amostra, faixa etária, percentual de mulheres na amostra, características particulares da amostra), metodológicas (e. g., domínios da AF, instrumento de avaliação da AF, instrumento de avaliação das barreiras, método de análise) e resultados (e. g., barreiras mencionadas ou com associações positivas). A partir dos dados da planilha de extração, elaborou-se a síntese descritiva.

Tomando como base a revisão prévia ${ }^{13}$, as barreiras identificadas nos artigos originais foram classificadas em três domínios: intrapessoais (e. g., relacionados aos interesses e possibilidades da pessoa em si, como, por exemplo, motivação, vontade e dor), interpessoais (e. g., relacionado ao contexto social, como os aspectos relacionamentais com família/amigos e demandas da ocupação) e ambientais (e. g., relacionados à infraestrutura disponível e à segurança do ambiente, como, por exemplo, existência de parques/facilidades e asfaltamento de ruas e percepção de risco de violência em um determinado bairro/local).

Em vista das distintas abordagens e tipos de análise utilizados para identificação das barreiras para a $\mathrm{AF}$ entre os artigos, definiu-se previamente que seriam apresentados na síntese descritiva: (I) nos artigos de abordagem qualitativa e/ou abordagem quantitativa descritiva: as barreiras respondidas pela maioria da amostra e (II) nos artigos quantitativos analíticos: as barreiras estatisticamente associadas à AF (e. g., considerando a mag- 
nitude da associação e o seu respectivo valor p). Para evitar comparações inadequadas destes dados, visto sua distinção, desde o momento da extração os resultados originais foram estratificados pelos seus tipos de análise (e. g., frequências, univariadas e multivariadas). Também, em vista da perspectiva "de escopo", não se avaliou o risco de viés dos artigos incluídos.

\section{Resultados}

As buscas conduzidas nas sete bases de dados eletrônicas e nas listas de referências recuperaram 911 artigos potenciais (907 pelas bases de dados e quatro nas buscas manuais) (Figura 1). Após identificação e exclusão de 96 registros duplicados, 815 artigos foram avaliados pelos seus títulos e resumos. Dos 95 que remanesceram no processo e foram encaminhados para avaliação pelos seus textos integrais, foram excluídos 82 , tendo como principais motivos: amostra não continha a faixa etária de interesse $(n=30)$ e ausência da análise de barreiras para a prática de AF $(n=19)$. Dessa forma, 13 artigos

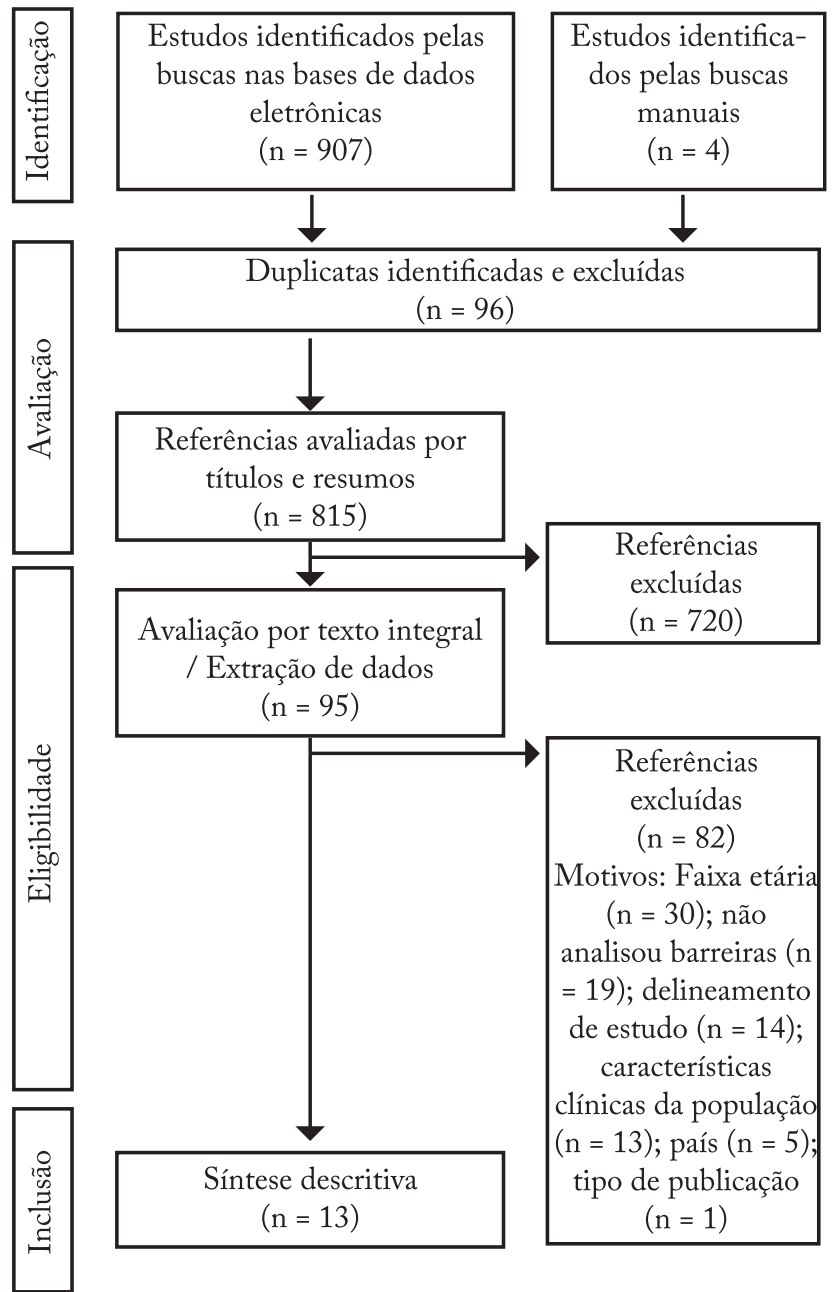

Figura 1 - Fluxograma da revisão de escopo originais responderam adequadamente aos critérios de inclusão e compuseram a síntese descritiva ${ }^{14-26}$.

Em relação aos objetivos, as pesquisas propuseram-se desde a identificação mais geral das barreiras para as $\mathrm{AF}^{14,16,18,21,23,24,26}$ às questões mais específicas, relacionadas à dor ${ }^{20}$, e à manutenção e/ou desistência de programas de $\mathrm{AF}^{15,25}$. Apenas o artigo de Gobbi et al. ${ }^{19}$ buscou identificar e analisar as barreiras associadas à inatividade física.

Geograficamente, as pesquisas foram conduzidas em cinco cidades diferentes de quatro estados brasileiros (Curitiba-Paraná, Florianópolis-Santa Catarina, Matelândia-Paraná, Pelotas-Rio Grande do Sul e Rio Claro-São Paulo) - Tabela 1. Os tamanhos amostrais variaram entre $10^{25}$ e $343^{24}$ idosos. Além de configurarem-se como a maioria em todas as amostras, cinco artigos foram conduzidos especificamente com amostras de mulheres ${ }^{16,20-22,26}$. Percebeu-se elevada heterogeneidade em relação às características dos participantes, destacando as amostras compostas por idosos partici-

Tabela 1 - Características descritivas dos estudos incluídos na síntese $(\mathrm{n}=13)$

\begin{tabular}{|c|c|c|c|}
\hline Referências & Local & $\begin{array}{c}\text { Amostra } \\
(\% \mathrm{~F})\end{array}$ & $\begin{array}{c}\text { Idade } \\
\text { (Características } \\
\text { particulares) }\end{array}$ \\
\hline Brazão et al. ${ }^{14}$ & $\begin{array}{l}\text { Rio Claro-São } \\
\text { Paulo }\end{array}$ & $79(58)$ & 71 (nd) \\
\hline Cardoso et al. ${ }^{15}$ & $\begin{array}{l}\text { Florianópolis- } \\
\text { Santa Catarina }\end{array}$ & $13(85)$ & $\begin{array}{c}72 \text { (Desistentes de um } \\
\text { programa de } \mathrm{AF} \text { ) }\end{array}$ \\
\hline Cassou et al. ${ }^{16}$ & $\begin{array}{l}\text { Curitiba- } \\
\text { Paraná }\end{array}$ & $25(100)$ & $\begin{array}{l}70 \text { (grupos de } \\
\text { convivência) }\end{array}$ \\
\hline Cavalli et al. ${ }^{26}$ & $\begin{array}{l}\text { Pelotas-Rio } \\
\text { Grande do Sul }\end{array}$ & $55(100)$ & $\begin{array}{c}\geq 60 \text { (Idosas participantes } \\
\text { de projeto) }\end{array}$ \\
\hline Eiras et al. ${ }^{17}$ & $\begin{array}{l}\text { Curitiba- } \\
\text { Paraná }\end{array}$ & $15(67)$ & $\geq 60$ (Praticantes de AF) \\
\hline Gobbi et al..$^{18}$ & $\begin{array}{l}\text { Rio Claro-São } \\
\text { Paulo }\end{array}$ & $30(63)$ & $\begin{array}{c}75 \text { (Idosos } \\
\text { institucionalizados) }\end{array}$ \\
\hline Gobbi et al. ${ }^{19}$ & $\begin{array}{l}\text { Rio Claro-São } \\
\text { Paulo }\end{array}$ & $359(63)$ & $\geq 60$ (nd) \\
\hline Krug et al. ${ }^{20}$ & $\begin{array}{l}\text { Florianópolis- } \\
\text { Santa Catarina }\end{array}$ & $30(100)$ & $\begin{array}{c}85 \text { (Inativas, grupos de } \\
\text { convivência) }\end{array}$ \\
\hline Krug et al. ${ }^{21}$ & $\begin{array}{l}\text { Florianópolis- } \\
\text { Santa Catarina }\end{array}$ & $89(100)$ & $\begin{array}{l}\geq 80 \text { (Inativas, grupos de } \\
\text { convivência) }\end{array}$ \\
\hline Lopes et al. ${ }^{22}$ & $\begin{array}{l}\text { Florianópolis- } \\
\text { Santa Catarina }\end{array}$ & $69(100)$ & $\begin{array}{l}\geq 80 \text { (Inativas, grupos de } \\
\text { convivência) }\end{array}$ \\
\hline Nascimento et al. ${ }^{23}$ & $\begin{array}{l}\text { Rio Claro-São } \\
\text { Paulo }\end{array}$ & $129(60)$ & 72 (nd) \\
\hline Oliveira et al..$^{24}$ & $\begin{array}{l}\text { Matelândia- } \\
\text { Paraná }\end{array}$ & 343 (nd) & $\begin{array}{c}\text { nd (Usuários das } \\
\text { Unidades Básicas de } \\
\text { Saúde) }\end{array}$ \\
\hline $\begin{array}{l}\text { Souza; } \\
\text { Vendrusculo }\end{array}$ & $\begin{array}{l}\text { Curitiba- } \\
\text { Paraná }\end{array}$ & 10 (nd) & $\begin{array}{c}\text { nd (Idosos participantes } \\
\text { de projeto) }\end{array}$ \\
\hline
\end{tabular}


pantes de grupos de convivência ${ }^{16,20-22}$, institucionaliza$\operatorname{dos}^{18}$ e participantes de projetos ${ }^{17,25,26}$

$\mathrm{Na}$ Tabela 2, observa-se que seis artigos foram desenvolvidos com abordagens quantitativas para coleta e análise de dados ${ }^{14,18,19,23,24,26}$, cinco artigos com abordagens qualitativas ${ }^{15,17,20,21,25}$ e dois com abordagens mis$\operatorname{tas}^{16,22}$. À exceção dos artigos de Nascimento et al. ${ }^{23}$ que buscou verificar barreiras à $\mathrm{AF}$ especificamente no tempo de lazer e de dois artigos com informações não disponíveis ${ }^{20,25}$ todas as pesquisas buscaram identificar barreiras para a AF em todos os domínios (deslocamento, doméstico, lazer e ocupacional).

Diversos instrumentos foram utilizados para avaliação da $\mathrm{AF}$, tendo maior frequência a versão longa do Questionário Internacional de Atividades Físicas (IPAQ) $(n=4)^{19,21,22,26}$ e os questionários próprios $(\mathrm{n}=3)^{14,18,16}$. Para identificação das barreiras, podem ser destacadas a técnica de grupos focais $(\mathrm{n}=5)$, com aplicação de roteiros estruturados ${ }^{20,21}$ semiestrutu$\operatorname{rados}^{25}$, ficha diagnóstica ${ }^{22}$ e a utilização de três ins-

Tabela 2 - Informações sobre os métodos utilizados nos artigos incluídos $(\mathrm{n}=13)$

\begin{tabular}{|c|c|c|}
\hline Referencias & $\begin{array}{c}\text { Instrumentos utilizados } \\
\text { para medida da } \mathrm{AF} / \\
\text { barreiras para } \mathrm{AF}\end{array}$ & $\begin{array}{c}\text { Métodos utilizados para } \\
\text { análise }\end{array}$ \\
\hline \multicolumn{3}{|c|}{ Artigos com coleta e análise de dados qualitativa } \\
\hline Cardoso et $\mathrm{al}^{15}$ & Entrevista semiestruturada & Análise descritiva \\
\hline Eiras et al. ${ }^{17}$ & Entrevista semiestruturada & $29-31$ \\
\hline Krug et al. ${ }^{20}$ & $\begin{array}{l}\text { QDE / Roteiro } \\
\text { estruturado* }\end{array}$ & Análise de conteúdo \\
\hline Krug et al. ${ }^{21}$ & $\begin{array}{c}\text { IPAQ (L) / Roteiro } \\
\text { estruturado* }\end{array}$ & Análise de conteúdo \\
\hline $\begin{array}{l}\text { Souza; } \\
\text { Vendrusculo }\end{array}$ & $\begin{array}{c}\text { Entrevista } \\
\text { semiestruturada* }\end{array}$ & 29,30 \\
\hline \multicolumn{3}{|c|}{ Artigos com coleta e análise de dados quantitativa } \\
\hline Cavalli et al. ${ }^{26}$ & IPAQ (L adaptado) $)^{27} / 28$ & Análise descritiva \\
\hline Brazão et al. ${ }^{14}$ & QDE / QBPAFI & Teste Qui-quadrado \\
\hline Gobbi et al. ${ }^{18}$ & $\mathrm{QDE} / 28$ & Análise descritiva \\
\hline Gobbi et al. ${ }^{19}$ & IPAQ (L) / QBPAP & Regressão Logística \\
\hline $\begin{array}{l}\text { Nascimento } \\
\text { et al. }{ }^{23}\end{array}$ & $\begin{array}{l}\text { Questionário de Baecke } \\
\text { adaptado / QBPAFI }\end{array}$ & Teste Qui-quadrado \\
\hline Oliveira et al. ${ }^{24}$ & $\mathrm{IPAQ}(\mathrm{C}) / \mathrm{QDE}$ & Regressão Logística \\
\hline \multicolumn{3}{|c|}{ Artigos com coleta e análise de dados mista } \\
\hline Cassou et al. ${ }^{16}$ & QDE / QDE aplicado* & $\begin{array}{c}\text { Análises de conteúdo e } \\
\text { descritiva }\end{array}$ \\
\hline Lopes et al. ${ }^{22}$ & $\begin{array}{l}\text { IPAQ }(\mathrm{L}) / \text { Ficha } \\
\text { diagnóstica* }\end{array}$ & Análise de conteúdo \\
\hline
\end{tabular}

* = grupo focal $\mathrm{AF}$ = atividade física $\mathrm{C}=$ versão curta; $\mathrm{IPAQ}=$ Questionário Internacional de Atividades Físicas; L = versão longa; QBPAFI $=$ Questionário de Barreiras à Prática de Atividade Física para Idosos; QBPAP = Questionário de Barreiras à Prática de Atividade Física; QDE = questionário desenvolvido para o estudo. trumentos prévios, em quatro artigos: o Questionário de Barreiras à Prática de Atividade Física para Idosos (QBPAFI $)^{14,23}$, o Questionário de Barreiras à Prática de Atividade Física (QBPAP) ${ }^{19}$ e o questionário sobre

Tabela 3 - Barreiras para atividade física identificadas entre os artigos, organizadas por domínios e tipos de análise

\begin{tabular}{|c|c|c|c|}
\hline \multirow{2}{*}{ Barreiras } & \multicolumn{3}{|c|}{ Tipo de análise } \\
\hline & Descritiva & Univariada & Multivariada \\
\hline \multicolumn{4}{|c|}{ Barreiras Intrapessoais $(\mathrm{n}=18)$} \\
\hline Alto Custo/Renda & $16(\mathrm{BR}), 8$ & 24 & \\
\hline AF não é prioridade & 21 & & \\
\hline Barreiras ao exercício & $16(\mathrm{BR})$ & & \\
\hline Doença, dor ou lesão & $\begin{array}{c}16(\mathrm{AR}), 17 \\
18,20,21 \\
22,25\end{array}$ & 14,23 & 19 \\
\hline $\begin{array}{l}\text { Falta de disposição/energia/ } \\
\text { motivação }\end{array}$ & 16(AR), 21 & 24 & \\
\hline Falta de persistência & & 23 & \\
\hline Falta de tempo & $16(\mathrm{BR})$ & & \\
\hline Jornada de trabalho extensa & $16(\mathrm{BR})$ & 24 & \\
\hline Limitação física & $\begin{array}{c}16(\mathrm{AR} / \mathrm{BR}) \\
21\end{array}$ & & \\
\hline Maneira de ser & 22 & & \\
\hline Medo de cair/se machucar & 21 & 14 우 & 19 \\
\hline Muito tímido & & 14 & \\
\hline Não gostar/Relação negativa & 21,22 & & \\
\hline
\end{tabular}
com AF

Necessidade de descanso

Nunca ter feito AF no lazer

Sentir-se suficientemente ativo

21

Sentir-se velho demais

14,23

Tarefas domésticas

21

14

16(BR)

\begin{tabular}{lc}
\hline \multicolumn{2}{c}{ Barreiras Interpessoais $(\mathrm{n}=8)$} \\
\hline Custo & $16(\mathrm{BR})$ \\
Excesso de cuidado da família & 21 \\
Exercícios físicos inadequados & 21 \\
Falta de apoio social/ & $16(\mathrm{AR})$, \\
companhia & 18,21 \\
Falta de rede social & $16(\mathrm{BR})$ \\
Obstáculos cotidianos & $16(\mathrm{AR} / \mathrm{BR})$ \\
Papel familiar & $21,22,25$ \\
Problemas de saúde do & 15 \\
cônjuge/demais familiares & Barreiras Ambientais $(\mathrm{n}=5)$ \\
\hline \multicolumn{2}{c}{$16(\mathrm{AR})$} \\
\hline Características ambientais & $16 \mathrm{AR}, 26$ \\
Condições climáticas & 18 \\
Falta de instalações perto de casa & $16(\mathrm{BR}), 18,21 \quad 149,23,24$ \\
Falta de segurança & 22 \\
Influência do meio-ambiente &
\end{tabular}

$\phi=$ Barreira em que respondentes do sexo feminino perceberam significativamente mais que o sexo masculino; $\mathrm{AF}$ = atividade física; $\mathrm{AR}=$ estrato de pessoas com alta renda: $\mathrm{BR}=$ estrato de pessoas com baixa renda. 
barreiras proposto por Hirayama ${ }^{28}$ em Gobbi et al. ${ }^{18}$ (Tabela 3). No que concerne às análises, dois artigos de abordagem qualitativa ${ }^{20,21}$ utilizaram análises de conteúdo e outros dois artigos ${ }^{17,25}$ os referenciais da pesquisa interpretativa ${ }^{29}$ e da "grounded theory" $30,31$. Nos artigos que se utilizaram da abordagem quantitativa, observou-se a utilização dos testes de associação qui-quadrado ${ }^{14,23}$ e regressões logísticas ${ }^{19,24}$ para identificação de associações e, nos artigos com abordagem mista, a utilização de análises de conteúdo ${ }^{16,22}$ e análise descritiva ${ }^{16}$

Ao todo, 31 barreiras para a AF foram identificadas e incluídas na síntese (Tabela 3). De acordo com a classificação proposta por Rech et al. ${ }^{13}$, as barreiras intrapessoais foram as mais frequentemente mencionadas entre os artigos incluídos $(\mathrm{n}=18 ; 60 \%)$. Mais especificamente, a variável "doença, dor ou lesão" $(n=10)$ foi a mais mencionada enquanto barreira para AF entre as análises descritivas $(\mathrm{n}=7)^{16-18,20-22,25}$ univariadas $(\mathrm{n}=$ $2)^{14,23}$, assim como associada à inatividade física na análise multivariada $(\mathrm{n}=1)$ apresentada por Gobbi et $\mathrm{al}^{(19)}$. Também, pela elevada frequência nas análises descritivas e associações nas análises uni e multivariadas, outras variáveis também merecem destaque, como "falta de segurança" (domínio ambiental; $\mathrm{n}=6$ ) 14,16,18,21,23,24; "medo de cair/se machucar" (domínio intrapessoal; $n$ $=3)^{14,19,21}$ e "sentir-se suficientemente ativo" (domínio intrapessoal; $n=3)^{14,19,23}$.

\section{Discussão}

A partir dos dados de 13 artigos originais, a presente revisão apontou em sua síntese a predominância de barreiras intrapessoais para a prática de $\mathrm{AF}$ entre idosos. Contudo, visto que a $\mathrm{AF}$ é determinada por distintos fatores sociais, econômicos e ambientais, é prudente evitar um entendimento simplista, onde as limitações para a prática de AF estão mais centradas nos indivíduos, de modo que a responsabilidade em se tornarem ativos depende, via de regra, da sua motivação, ou mesmo do interesse em se manterem saudáveis. Outros artigos conduzidos no país ${ }^{32,33}$, mas que não adotam a terminologia "barreiras", sugerem que distintos fatores, para além dos intrapessoais, estão atrelados à prática de $\mathrm{AF}$ e, dessa forma, devem ser levados em consideração no debate, assim como nos processos de elaboração e implementação de ações de promoção da AF em idosos.

A presente revisão também destaca que a barreira intrapessoal "doença, dor ou lesão" foi reportada em dez artigos originais. Mais especificamente, ela foi mencionada em sete artigos descritivos ${ }^{16-18,20-22,25}$, esteve associada à $\mathrm{AF}$ em duas análises univariadas ${ }^{12,23}$ e associada à inatividade física em uma análise multivariada $^{19}$. Esta barreira pode ser discutida por distintos aspectos: em relação ao aspecto biológico, artigos conduzidos no país indicam alta prevalência de dor crônica em idosos ${ }^{34}$ principalmente nos membros inferiores ${ }^{35}$ e na região dorsal/lombar ${ }^{36}$, sendo apontados maiores riscos em mulheres ${ }^{34,35}$ pessoas com idade avançada ${ }^{34}$, pessoas com pior percepção de saúde ${ }^{35}$ e pessoas de baixa renda ${ }^{34}$. Por outro lado, é também possível de se hipotetizar que as dores relacionadas à prática de $\mathrm{AF}$ se coloquem como um limitador à sua prática, na qualidade de efeito direto.

Ao discutido, se soma a barreira "medo de cair/se machucar", mencionada em três artigos incluídos na síntese ${ }^{14,19,21}$. Ao reconhecer que o medo pode desempenhar um papel preditivo mais significativo à não-realização de $\mathrm{AF}$ do que as próprias características da dor $^{37}$, da mesma forma que é importante um acompanhamento das dores já existentes nos idosos, cabe pontuar sobre a necessidade de uma orientação mais abrangente para a prática, que possa, para além da informação sobre os benefícios, buscar dirimir os medos e dar o adequado suporte às eventuais dores que podem decorrer da prática de AF.

Em uma perspectiva geral, um ponto importante a ser ponderado é a relação entre as barreiras mais relatadas à prática de AF e barreiras que estão associadas à inatividade física. Apenas o artigo de Gobbi et al. ${ }^{19}$ identificou que as barreiras "doença, dor ou lesão", "medo de cair/se machucar" e "sentir-se suficientemente ativo" estão associadas à inatividade física. É prudente pontuar que nem sempre a barreira mais reportada para a prática de $\mathrm{AF}$ está associada à inatividade física, podendo-se sugerir sobre a importância de futuros estudos, que caminhem para além da identificação das principais barreiras à $\mathrm{AF}$, analisando também sua possível associação à inatividade física nos idosos brasileiros.

A segunda barreira mais mencionada entre os artigos incluídos foi "falta de segurança"14,16,18,21,23,24, pertencente ao domínio ambiental. Para além da consideração que esta variável foi reportada em idosos de quatro cidades de diferentes portes (e. g., Curitiba-Paraná, Florianópolis-Santa Catarina, Matelândia-Paraná e Rio Claro-São Paulo), vale destacar que em dois artigos esta barreira foi mais percebida por pessoas de baixa renda ${ }^{16}$ e por mulheres ${ }^{14}$. Nesse sentido, torna-se interessante aprofundar o respectivo motivo (ou 
motivos), se por risco de acidentes, quedas (e. g., pela falta de pavimentação, trânsito, ou mesmo características acidentadas dos bairros), ou mesmo por questões de violência e criminalidade. $\mathrm{O}$ conhecimento prévio destaca que ruas escuras ${ }^{38}$, violência ${ }^{39}$, características do trânsito $^{40}$ e medo de ser assaltado ${ }^{33}$ se constituem como fatores limitadores para a prática de $\mathrm{AF}$ no país e isso reforça que uma boa percepção de segurança é essencial para a realização de $\mathrm{AF}$ ao ar livre, sobretudo no tempo de lazer e como forma de deslocamento.

Em muitas cidades brasileiras a população não se sente segura para frequentar os espaços públicos ${ }^{41}$ (e. g., praças, academias ao ar livre e parques) mais adequados para prática de $\mathrm{AF}$ no lazer. Se os locais não são seguros, muitos idosos podem procurar outras formas de passar seu tempo livre, modificando assim suas opções ${ }^{42}$. Maiores investimentos em iluminação pública e no reforço de segurança pública nestes espaços, assim como no seu entorno podem ser alternativas para alteração deste cenário.

No mesmo sentido, a preocupação com locais adequados, sejam espaços ao ar livre ou ambientes fechados, para que idosos se sintam seguros e motivados para a prática de AF, pode auxiliar na superação destas barreiras, bem como, melhor informá-los sobre as possibilidades que a prática regular de $\mathrm{AF}$ pode promover para sua saúde e qualidade de vida. Conjuntamente a isso, promover atividades que estimulem a interação entres os idosos e aqueles que lhe estão próximos pode, além de reforçar os vínculos, estimular a prática de $\mathrm{AF}$ e adoção de hábitos saudáveis entre mais pessoas. As barreiras interpessoais "Falta de apoio social/companhia"16,18,21 e "Papel familiar" 21,22,25 reforçam esta sugestão.

A percepção de "sentir-se suficientemente ativo" ${ }^{14,19,23}$, configurou-se como terceira barreira mais frequentemente mencionada para a prática de AF entre as pesquisas disponíveis. Vale ponderar que todos os estudos que sugerem esta barreira foram conduzidos na cidade de Rio Claro-São Paulo, com uso de distintos indicadores para classificar "AF suficiente" (e. g., prática de $\mathrm{AF}$ regular realizada na maioria dos dias da semana, pelo menos 30 minutos por dia ${ }^{14}$; pelo menos 150 minutos semanais, com períodos de, pelo menos 10 minutos de prática $\left.{ }^{19}\right)$. De qualquer forma, pode-se hipotetizar que a percepção de se sentir suficientemente ativo esteja atrelada às atividades da vida diária, que, na avaliação dos respondentes poderiam suprir, adequadamente, as quantidades de AF apontadas pelos estudos. Entretanto, reforça-se a importância das AF no tempo de lazer, não apenas para a melhoria ou manutenção de indicadores metabólicos, mas para outros fins, como o prazer e a socialização.

É profícuo apontar a limitação regional dos estudos, a partir da identificação que quatro das cinco cidades com dados disponíveis situam-se na região Sul do país. Dessa forma, ao mesmo tempo que se recomenda cautela na extrapolação dos dados apresentados - visto que não foram incluídos estudos conduzidos em cidades das regiões Centro-Oeste, Nordeste e Norte do Brasil - se ressalta a importância de estudos conduzidos em cidades destas regiões, de modo que estratégias mais específicas possam ser elaboradas a partir das distintas realidades. Complementarmente, ao mencionar que apenas três dos artigos envolvidos na síntese foram publicados nos últimos cinco $\operatorname{anos}^{22,24,26}$, recomenda-se a condução de pesquisas mais recentes, visto a ampliação de espaços de oferta de $\mathrm{AF}$ utilizados por adultos e idosos, como as academias ao ar livre $^{43}$ e o Programa Academia da Saúde ${ }^{44}$.

Também cabe destacar uma outra limitação, visto que, de acordo com os critérios de inclusão, apenas foram incluídos artigos que adotaram a terminologia "barreiras". Não foram considerados para a síntese, por exemplo, artigos que investigaram fatores associados e determinantes da inatividade física. Por fim, os artigos incluídos na revisão foram, em sua maioria, desenvolvidos com amostras não-probabilísticas por conveniência e intencional, logo, os resultados podem não refletir as barreiras de idosos com características distintas.

Concluindo, identificou-se por meio deste trabalho que as barreiras intrapessoais são as mais percebidas pelos idosos brasileiros, sendo a barreira "dor, doença e/ou lesão" a mais frequentemente mencionada, seguida das barreiras "falta de segurança"; "medo de cair/se machucar" e "sentir-se suficientemente ativo". Mesmo que esta evidência esteja amparada, em sua maior parte, por dados provenientes da região Sul do país, o reconhecimento destas barreiras pode ser incorporada nos distintos cenários de oferta da AF para idosos. Complementarmente, futuros estudos são importantes, tanto para apresentação das principais barreiras para a $\mathrm{AF}$ nas distintas regiões do país, quanto para a análise das suas possíveis associações à inatividade física.

\section{Conflito de interesse}

Os autores declaram não haver conflito de interesse.

\section{Contribuição dos autores}

Socoloski TS e Guerra PH, participaram da concepção da ideia 
central do estudo, elaboração das buscas sistemáticas, avaliação dos títulos, resumos e textos integrais, desenvolvimento da síntese, análise dos resultados e escrita do texto. Rech CR e Hino AAF, participaram da análise dos resultados e revisão do texto. Correa Junior JA e Lopes RM, participaram da avaliação dos títulos, resumos e textos integrais, desenvolvimento da síntese, análise dos resultados e revisão do texto.

\section{Referências}

1. Vasconcelos AMN, Gomes MMF. Transição demográfica: a experiência brasileira. Epidemiol Serv Saúde.2012;21(4):53948.

2. Nunes BP, Batista SRR, Andrade FB, Souza Junior PRB, Lima-Costa MF, Facchini LA. Multimorbidity: The Brazilian Longitudinal Study of Aging (ELSI-Brazil). Rev Saúde Pública. 2018;52(Suppl 2),10s.

3. Alves LC, Leite IC, Machado CJ. Fatores associados à incapacidade funcional dos idosos no Brasil: análise multinível. Rev Saúde Pública. 2010;44(3):468-78.

4. World Health Organization, US National Institute of Aging. Global Health and Ageing. Geneva: World Health Organization; 2011.

5. Alcântara AA, Camarano AA, Giacomin KC. Política nacional do idoso: velhas e novas questões. Rio de Janei-ro: Ipea; 2016.

6. Ueno DT, Gobbi S, Teixeira CVL, Sebastião E, Prado AKG, Costa JLR, Gobbi LTB. Efeitos de três modali-dades de atividade física na capacidade funcional de idosos. Rev Bras Educ Fís Esporte. 2012;26(2):273-81.

7. Northey JM, Cherbuin N, Pumpa KL, Smee DJ, Rattray B. Exercise interventions for cognitive function in adults older than 50: a systematic review with meta-analysis. Br J Sports Med. 2018;52(3):154-60.

8. Beck AP, Antes DL, Meurer ST, Benedetti TRB, Lopes MA. Fatores associados às quedas entre idosos prati-cantes de atividades físicas. Texto Contexto - Enferm. 2011;20(2):28086.

9. Ribeiro LHM, Neri AL. Exercícios físicos, força muscular e atividades de vida diária em mulheres idosas. Ci-ênc Saúde Coletiva. 2012;17(8):2169-80.

10. Silva MF, Goulart NBA, Lanferdini FJ, Marcon M, Dias CP. Relação entre os níveis de atividade física e qua-lidade de vida de idosos sedentários e fisicamente ativos. Rev Bras Geriatr Gerontol. 2012;15(4):634-42.

11. Latorre-Román PA, Laredo-Aguilera JA, García-Pinillos F, Soto-Hermoso VM, Carmona-Torres JM. Physical activity, weight and functional limitations in elderly Spanish people: the National Health Survey (2009-2014). Eur J Public Health. 2018;28(4):778-83.

12. Cruz MS, Bernal RTI, Claro RM. Tendência da prática de atividade física no lazer entre adultos no Brasil (2006-2016). Cad Saúde Pública. 2018;34(10),e00114817.

13. Rech CR, Camargo EM, Araujo PAB, Loch MR, Reis RS. Perceived barriers to leisure-time physical activity in the Brazilian population. Rev Bras Med Esporte. 2018;24(4):303-9.

14. Brazão MC, Hirayama MS, Gobbi S, Nascimento CMC, Roseguini AZ. Estágios de mudança de comporta-mento e barreiras percebidas à prática de atividade física em idosos residentes em uma cidade de médio porte do Brasil. Motriz. 2009;15(4):759-67.
15. Cardoso AS, Borges LJ, Mazo GZ, Benedetti TB, Kuhnen AP. Fatores influentes na desistência de idosos em um programa de exercício físico. Movimento. 2008;14(1):225-39.

16. Cassou AC, Fermino R, Rodriguez Añez CR, Santos MS, Domingues MR, Reis RS. Barriers to physical activ-ity among Brazilian elderly women from different socioeconomic status: a focus-group study. J Phys Act Health. 2011;8(1):126-32.

17. Eiras SB, Silva WHA, Souza DL, Vendruscolo R. Fatores de adesão e manutenção da prática de atividade físi-ca por parte de idosos. Rev Bras Cienc Esporte. 2010;31(2):75-89.

18. Gobbi S, Caritá LP; Hirayama MS; Quadros Junior AC; Santos RF, Gobbi LTB. Comportamento e barreiras: atividade física em idosos institucionalizados. Psicol Teor Pesq. 2008;24(4):451-8.

19. Gobbi S, Sebastião E, Papini CB, Nakamura PM, Valdanha Netto A, Gobbi LT, Kokubun E. Physical inac-tivity and related barriers: a study in a community dwelling of older brazilians. J Aging Res. 2012;2012:685190.

20. Krug RR, Lopes MA, Mazo GZ, Marchesan M. A dor dificulta a prática de atividade física regular na percep-ção de idosas longevas. Rev Dor. 2013;14(3),192-5.

21. Krug RR, Lopes MA, Mazo GZ. Barreiras e facilitadores para a prática da atividade física de longevas inativas fisicamente. Rev Bras Med Esporte. 2015;21(1),57-64.

22. Lopes MA, Krug RR, Bonetti A, Mazo GZ. Barreiras que influenciaram a não adoção de atividade física por longevas. Rev Bras Ciênc Esporte. 2016;38(1):76-83.

23. Nascimento CMC, Gobbi S, Hirayama MS, Brazão MC. Nivel de atividade física e as principais barreiras per-cebidas por idosos de Rio Claro. Rev Educ Fís. 2008;19(1):109-18.

24. Oliveira DV, Brito RL, Antunes MD; Nascimento Júnior JRA, Moreira CR; Oliveira DC, Lima MCC; Berto-lini SMMG, Cavaglieri CR. Prática de atividade física por idosos frequentadores de unidades básicas de saúde. Geriatr Gerontol Aging 2017; 11(3): 116-23.

25. Souza DL, Vendrusculo R. Fatores determinantes para a continuidade da participação de idosos em programas de atividade física: a experiência dos participantes do projeto "Sem Fronteiras”. Rev Bras Educ Fís Esporte. 2010;24(1):95-105.

26. Mazo GZ, Benedetti TRB. Adaptação do questionário internacional de atividade física para idosos. Rev Bras Cineantropom Desempenho Hum. 2010;12(6):480-4.

27. Cavalli AS, Mello DM, Cavalli LS, Colpo GS, Arruda STC, Meireles LG. Barreiras para a atividade física em mulheres idosas do sul do Brasil. Pixo 2020;13(4):209-19.

28. Hirayama, MS. Atividade física e doença de Parkinson: mudança de comportamento, autoeficácia, barreiras percebidas e qualidade de vida. [Dissertação de Mestrado]. Rio Claro: Universidade Estadual Paulista (Unesp); 2006.

29. Geertz C. A interpretação das culturas. Rio de Janeiro: LTC; 1989.

30. Glaser BG, Strauss AL. The discovery of grounded theory: strategies for qualitative research. Chicago: Aldine; 1967.

31. Strauss AL, Corbin J. Introduction to qualitative research: grounded theory procedures and techniques. Bever-ly Hills: Sage; 1990.

32. Peixoto SV, Mambrini JVM, Firmo JOA, Loyola Filho AI, Souza Junior PRB, Andrade FB, Lima-Costa MF. Physical activity practice among older adults: results of the ELSIBrazil. Rev Saude Publica. 2018;52(Suppl 2):5s.

33. Giehl MWC, Schneider IJC, Corseuil HX, Benedetti TRB, d'Orsi E. Atividade física e percepção do ambien-te em idosos: estudo populacional em Florianópolis. Rev Saúde Pública. 2012;46(3):516-25. 
34. Carvalho RC, Maglioni CB, Machado GB, Araújo JE, Silva JRT, Silva ML. Prevalence and characteristics of chronic pain in Brazil: a national internet-based survey study. BrJP. 2018;1(4):331-8.

35. Pereira LV, Vasconcelos PP, Souza LAF, Pereira GA, Nakatani AYK, Bachion MM. Prevalence and intensity of chronic pain and self-perceived health among elderly people: a population-based study. Rev Latino-Am En-fermagem. 2014;22(4):662-9.

36. Vasconcelos FH, Araújo GC. Prevalence of chronic pain in Brazil: a descriptive study. BrJP. 2018;1(2):176-9.

37. Larsson C, Ekvall Hansson E, Sundquist K, Jakobsson U. Impact of pain characteristics and fear-avoidance be-liefs on physical activity levels among older adults with chronic pain: a population-based, longitudinal study. BMC Geriatr. 2016;16:50.

38. Silva MC, Silva AB, Amorim TEC. Condições de espaços públicos destinados a prática de atividades Físicas na cidade de Pelotas/rS/Brasil. Rev Bras Ativ Fis Saúde. 2012;17(1):28-32.

39. Queiroga MR, Ferreira SA, Boneti MD, Tartaruga MP, Coutinho SS, Cavazzoto TG. Caracterização do am-biente físico e prática de atividades físicas em unidades básicas de saúde de Guarapuava, Paraná, 2011-2012. Epidemiol Serv Saúde. 2016;25(4):827-36.
40. Kretschmer AC, Dumith SC. Prática de atividade física no lazer e ambiente percebido: um estudo de base po-pulacional com adultos e idosos do Sul do Brasil. Rev Bras Epidemiol. 2020;23:e200043.

41. Aguiar JB, Nascimento INM, Melo MA, Freire AKU, Saraiva LC, Santos ALB, Gurgel LA. Espaços públicos de lazer de uma capital brasileira: avaliação da qualidade e uso para a prática de atividade física. Licere. 2019,22(4):317-39.

42. Freire DRA, Oliveira EA, Rocha Junior CP. Práticas de lazer em Salvador: análise comparada entre dois bair-ros. Licere. 2019;22(3):159-88.

43. Battistel JA, Floss MI, Cruvinel AFP, Barbato PR, Fermino RC, Guerra PH. Perfil dos frequentadores e pa-drão de utilização das academias ao ar livre: revisão de escopo. Rev Bras Ativ Fís Saúde. 2021;26:e0186.

44. Guerra PH, Andrade DR, Rodriguez-Añez CR, Santos DL, Camargo EM, Fermino RC, Tenório MCM. Re-search on the Academia da Cidade and Academia da Saúde Programs: scoping review. Rev Bras Ativ Fís Saú-de. 2020;25:e0126.

Recebido: $24 / 02 / 2021$

Aprovado: 16/06/2021

\section{Como citar este artigo:}

Socoloski TS, Rech CR, Correa Junior JA, Lopes RM, Hino AAF, Guerra PH. Barreiras para a prática de atividade física em idosos: revisão de escopo de estudos brasileiros. Rev Bras Ativ Fis Saúde. 2021;26:e0208. DOI: 10.12820/rbafs.26e0208 